\title{
Alternating sums of the powers of Fibonacci and Lucas numbers
}

Emrah Kiliç, Neşe Ömür, and Yücel Türker Ulutaş 


\title{
ALTERNATING SUMS OF THE POWERS OF FIBONACCI AND LUCAS NUMBERS
}

\author{
EMRAH KILIÇ, NEŞE ÖMÜR, AND YÜCEL TÜRKER ULUTAŞ \\ Received 17 May, 2010
}

Abstract. We shall consider alternating Melham's sums for Fibonacci and Lucas numbers of the form $\sum_{k=1}^{n}(-1)^{k} F_{2 k+\delta}^{2 m+\varepsilon}$ and $\sum_{k=1}^{n}(-1)^{k} L_{2 k+\delta}^{2 m+\varepsilon}$, where $\varepsilon, \delta \in\{0,1\}$.

2000 Mathematics Subject Classification: 11B37; 11B39; 05A19

Keywords: Fibonacci and Lucas numbers, alternating sums, Binet formulas

\section{INTRODUCTION}

The Fibonacci $F_{n}$ and Lucas numbers $L_{n}$ are defined by the following recursions: for $n>0$,

$$
F_{n+1}=F_{n}+F_{n-1} \text { and } L_{n+1}=L_{n}+L_{n-1},
$$

where $F_{0}=0, F_{1}=1$ and $L_{0}=2, L_{1}=1$, respectively.

If the roots of the characteristic equation $x^{2}-x-1=0$ are $\alpha$ and $\beta$, then the Binet formulas are

$$
F_{n}=\frac{\alpha^{n}-\beta^{n}}{\alpha-\beta} \text { and } L_{n}=\alpha^{n}+\beta^{n} .
$$

Wiemann and Cooper [6] raised certain conjectures for the Melham sum:

$$
\sum_{k=1}^{n} F_{2 k}^{2 m+1} .
$$

Ozeki [2] considered Melham's sum and he gave an explicit expansion for it as a polynomial in $F_{2 n+1}$.

More generally, Prodinger [3] derived a formula for the sum:

$$
\sum_{k=0}^{n} F_{2 k+\delta}^{2 m+\varepsilon},
$$

where $\varepsilon, \delta \in\{0,1\}$. He also evaulated the corresponding sums for the Lucas numbers. 
In this paper, we consider the alternating analogs of Melham's sums. We derive explicit formulas for the sums:

$$
\sum_{k=1}^{n}(-1)^{k} F_{2 k+\delta}^{2 m+\varepsilon} \text { and } \sum_{k=1}^{n}(-1)^{k} L_{2 k+\delta}^{2 m+\varepsilon},
$$

where $\varepsilon, \delta \in\{0,1\}$.

\section{Alternating Melham's Sums for FibonacCi numbers}

In this section we will start with some lemmas and then we shall derive our results about the alternating Melham's sum.

Lemma 1. For positive integers $n, m$ and $t$ such that $m \geq t$,

i) $(-1)^{t+1} F_{(2 m-2 t+1) n}+F_{(2 m-2 t+1)(n+1)}$

$$
=\sum_{j=0}^{2 m-2 t}(-1)^{j(t-2)} F_{(2 m-2 t+1) n+j+\left(1-3(-1)^{t}\right) / 2},
$$

and

ii) $F_{2(m-t)(n+1)}-(-1)^{t} F_{2(m-t) n}= \begin{cases}F_{2(m-t) n+m-t} L_{m-t} & \text { if } m \text { is odd, } \\ L_{2(m-t) n+m-t} F_{m-t} & \text { if } m \text { is even. }\end{cases}$

Proof. $i$ ) We can write

$$
\begin{aligned}
& (-1)^{t+1} F_{(2 m-2 t+1) n}+F_{(2 m-2 t+1)(n+1)} \\
& =(-1)^{t+1}\left(\frac{\alpha^{(2 m-2 t+1) n}-\beta^{(2 m-2 t+1) n}}{\alpha-\beta}\right) \\
& +\left(\frac{\alpha^{(2 m-2 t+1)(n+1)}-\beta^{(2 m-2 t+1)(n+1)}}{\alpha-\beta}\right) \\
& =\frac{\alpha^{(2 m-2 t+1) n}\left((-1)^{t+1}+\alpha^{2 m-2 t+1}\right)}{\alpha-\beta} \\
& -\frac{\beta^{(2 m-2 t+1) n}\left((-1)^{t+1}+\beta^{2 m-2 t+1}\right)}{\alpha-\beta} \\
& = \begin{cases}\sum_{j=0}^{2 m-2 t}(-1)^{j}\left(F_{(2 m-2 t+1) n+j}+F_{(2 m-2 t+1) n+j+1}\right) & \text { if } t \text { is odd, } \\
-\sum_{j=0}^{2 m-2 t} F_{(2 m-2 t+1) n+j}-F_{(2 m-2 t+1) n+j+1} & \text { if } t \text { is even, }\end{cases}
\end{aligned}
$$




$$
= \begin{cases}\sum_{j=0}^{2 m-2 t}(-1)^{j} F_{(2 m-2 t+1) n+j+2} & \text { if } t \text { is odd, } \\ \sum_{j=0}^{2 m-2 t} F_{(2 m-2 t+1) n+j-1} & \text { if } t \text { is even, } \\ =\sum_{j=0}^{2 m-2 t}(-1)^{j(t-2)} F_{(2 m-2 t+1) n+j+\left(1-3(-1)^{t}\right) / 2} .\end{cases}
$$

ii) The proof is similar to the ones for the Binet formulas of $\left\{F_{n}\right\}$ and $\left\{L_{n}\right\}$.

From [4], we have the following result for the Gibonacci sequence $\left\{G_{n}\right\}$, defined by for $n>0$

$$
G_{n+1}=G_{n}+G_{n-1},
$$

with arbitrary initial values $G_{0}$ and $G_{1}$.

Lemma 2. Let $a, p \neq 0, q$ be arbitrary integers. Then for $n>0$,

$$
\sum_{i=a}^{n} G_{p i+q}=\frac{G_{p(n+1)+q}+(-1)^{p+1} G_{p n+q}+(-1)^{p} G_{p(a-1)+q}-G_{p a+q}}{L_{p}-1-(-1)^{p}},
$$

and

$$
\begin{aligned}
& \sum_{i=a}^{n}(-1)^{i} G_{p i+q} \\
= & \frac{(-1)^{n} G_{p(n+1)+q}+(-1)^{p+n} G_{p n+q}+(-1)^{a} G_{p a+q}+(-1)^{a+p} G_{p(a-1)+q}}{1+(-1)^{p}+L_{p}} .
\end{aligned}
$$

As a consequence of Lemma 2, for further use we state the following result:

Corollary 1. For any integer $r$ and positive even integer $t$,

$$
\sum_{k=1}^{n}(-1)^{k r} L_{k t}=\frac{(-1)^{n r} F_{t(n+1)}+(-1)^{n r-r} F_{t n}-F_{t}}{F_{t}}
$$

and

$$
\sum_{k=1}^{n}(-1)^{k r} F_{k(t+1)}=\frac{(-1)^{(n+1) r} F_{(t+1) n}+(-1)^{n r} F_{(t+1)(n+1)}-F_{t+1}}{L_{t+1}} .
$$

Proof. Clearly

$$
\sum_{k=1}^{n}(-1)^{k r} L_{k t}=(-1)^{r} L_{t}+L_{2 t}+\ldots+(-1)^{n r} L_{n t} .
$$


For the first claim, we consider two cases: the first case is when $n$ is an odd integer. Here

$$
\sum_{k=1}^{n}(-1)^{k r} L_{k t}=(-1)^{r} \sum_{j=1}^{(n+1) / 2} L_{(2 j-1) t}+\sum_{j=1}^{(n-1) / 2} L_{2 j t} .
$$

If we take $a=1, p=2 t, q=-t$ and $n \rightarrow \frac{n+1}{2}$ in Lemma 2, then we get

$$
\sum_{j=1}^{(n+1) / 2} L_{(2 j-1) t}=\frac{L_{t(n+2)}-L_{t n}+L_{-t}-L_{t}}{L_{2 t}-2} .
$$

The following identities are well known [1,5]:

$$
L_{c+t}-L_{c-t}=5 F_{c} F_{t}
$$

for even $t$, and

$$
L_{2 c}-(-1)^{c} 2=5 F_{c}^{2} \text { and } L_{-c}=(-1)^{c} L_{c}
$$

for any integer $c$. Thus we have

$$
\sum_{j=1}^{(n+1) / 2} L_{(2 j-1) t}=\frac{5 F_{t(n+1)} F_{t}}{5 F_{t}^{2}}=\frac{F_{t(n+1)}}{F_{t}} .
$$

Meanwhile, if we take $a=1, p=2 t, q=0$ and $n \rightarrow \frac{n-1}{2}$ in Lemma 2, then we get

$$
\sum_{j=1}^{(n-1) / 2} L_{2 j t}=\frac{L_{t(n+1)}-L_{t(n-1)}+L_{0}-L_{2 t}}{L_{2 t}-2}
$$

Since $t$ is even, by (2.2) and (2.3), we rewrite the last equation as

$$
\sum_{j=1}^{(n-1) / 2} L_{2 j t}=\frac{5 F_{t n} F_{t}}{5 F_{t}^{2}}-1=\frac{F_{t n}}{F_{t}}-1 .
$$

If we sustitute (2.4) and (2.5) in (2.1), then we obtain

$$
\begin{aligned}
\sum_{k=1}^{n}(-1)^{k r} L_{k t} & =(-1)^{r}\left(\frac{F_{t(n+1)}}{F_{t}}\right)+\left(\frac{F_{t n}}{F_{t}}-1\right) \\
& =\frac{(-1)^{r} F_{t(n+1)}+F_{t n}-F_{t}}{F_{t}} .
\end{aligned}
$$

For the second case, let $n$ be an even integer, thus

$$
\sum_{k=1}^{n}(-1)^{k r} L_{k t}=(-1)^{r} \sum_{j=1}^{n / 2} L_{(2 j-1) t}+\sum_{j=1}^{n / 2} L_{2 j t} .
$$


By taking $a=1, p=2 t, q=-t$ and $n \rightarrow \frac{n}{2}$ and $a=1, p=2 t, q=0$ and $n \rightarrow \frac{n}{2}$ in Lemma 2, respectively, we obtain the following result by (2.2) and (2.3), for even $t$,

$$
\begin{aligned}
\sum_{j=1}^{n / 2} L_{(2 j-1) t} & =\frac{F_{t n}}{F_{t}} \\
\sum_{j=1}^{n / 2} L_{2 j t} & =\frac{F_{t(n+1)}}{F_{t}}-1 .
\end{aligned}
$$

If we put (2.8) and (2.9) in (2.7), we get

$$
\begin{aligned}
\sum_{k=1}^{n}(-1)^{k r} L_{k t} & =(-1)^{r}\left(\frac{F_{t n}}{F_{t}}\right)+\left(\frac{F_{t(n+1)}}{F_{t}}-1\right) \\
& =\frac{(-1)^{r} F_{t n}+F_{t(n+1)}-F_{t}}{F_{t}} .
\end{aligned}
$$

Combining (2.6) and (2.10), we get the final result:

$$
\sum_{k=1}^{n}(-1)^{k r} L_{k t}=\frac{(-1)^{n r} F_{t(n+1)}+(-1)^{n r-r} F_{t n}-F_{t}}{F_{t}},
$$

as claimed.

Finally by taking $a=1, p=t+1, q=0$ in Lemma 2, the second claim is obtained similary to the first claim.

Theorem 1. i) For positive odd $m$,

$$
\begin{aligned}
\sum_{k=1}^{n}(-1)^{k} F_{k}^{2 m}=\frac{1}{5^{m}} \sum_{i=0}^{m-1}(-1)^{i(n+1)+n}\left(\begin{array}{c}
2 m \\
i
\end{array}\right) \frac{F_{(m-i)(2 n+1)}}{F_{m-i}} & -\frac{1}{5^{m}}\left(\begin{array}{c}
2 m-1 \\
m
\end{array}\right)-\frac{1}{5^{m}}\left(\begin{array}{c}
2 m \\
m
\end{array}\right) n
\end{aligned}
$$

and, for positive even $m$,

$$
\begin{aligned}
& \sum_{k=1}^{n}(-1)^{k} F_{k}^{2 m} \\
= & \left\{\begin{array}{c}
\frac{1}{5^{m}}\left(\sum_{i=0}^{m-1}(-1)^{i}\left(\begin{array}{c}
2 m \\
i
\end{array}\right) \frac{L_{(m-i)(2 n+1)}}{L_{m-i}}+\left(\begin{array}{c}
2 m-1 \\
m
\end{array}\right)\right) \\
-\frac{1}{5^{m}}\left(\sum_{i=0}^{m-1}\left(\begin{array}{c}
2 m \\
i
\end{array}\right) \frac{L_{(m-i)(2 n+1)}}{L_{m-i}}+\left(\begin{array}{c}
2 m-1 \\
m-1
\end{array}\right)\right)
\end{array} \quad \text { if } n \text { is oden, },\right.
\end{aligned}
$$


and

$$
\text { ii) } \begin{aligned}
\sum_{k=1}^{n}(-1)^{k} F_{k}^{2 m+1}= & \frac{1}{5^{m}} \sum_{i=0}^{m}\left(\begin{array}{c}
2 m+1 \\
i
\end{array}\right) \frac{(-1)^{i(n+1)+n}}{L_{2 m-2 i+1}} \\
& \times \sum_{j=0}^{2(m-i)}(-1)^{j(i-2)} F_{n(2 m-2 i+1)+j+\frac{1-3(-1)}{2}} \\
& -\frac{1}{5^{m}} \sum_{i=0}^{m}(-1)^{i}\left(\begin{array}{c}
2 m+1 \\
i
\end{array}\right) \frac{F_{2 m-2 i+1}}{L_{2 m-2 i+1}} .
\end{aligned}
$$

Proof. $i$ ) For odd $m$, consider

$$
\begin{aligned}
& \sum_{k=1}^{n}(-1)^{k} F_{k}^{2 m} \\
= & \sum_{k=1}^{n}(-1)^{k}\left(\frac{\alpha^{k}-\beta^{k}}{\alpha-\beta}\right)^{2 m} \\
= & \sum_{k=1}^{n}(-1)^{k} \frac{1}{(\alpha-\beta)^{2 m}} \sum_{i=0}^{2 m}(-1)^{i}\left(\begin{array}{c}
2 m \\
i
\end{array}\right) \alpha^{k i} \beta^{k(2 m-i)} \\
= & \sum_{k=1}^{n} \frac{(-1)^{k}}{5^{m}}\left(\sum_{i=0}^{m}(-1)^{i}\left(\begin{array}{c}
2 m \\
i
\end{array}\right)\left(\alpha^{k(2 m-i)} \beta^{k i}+\alpha^{k i} \beta^{k(2 m-i)}\right)\right. \\
= & \frac{1}{5^{m}}\left(\begin{array}{c}
m-1 \\
\sum_{i=0}^{m}\left(\begin{array}{c}
2 m \\
m
\end{array}\right)(\alpha \beta)^{k m}
\end{array}\right) \\
& +\left(\begin{array}{c}
2 m \\
m
\end{array}\right) \sum_{k=1}^{n}(-1)^{m(k+1)+k}(2 m) \sum_{k=1}^{n}(-1)^{k(i+1)} L_{2 k(m-i)}
\end{aligned}
$$

By taking $i+1=r$ and $2(m-i)=t$ in Corollary 1 , we write

$$
\begin{aligned}
& \sum_{k=1}^{n}(-1)^{k} F_{k}^{2 m} \\
= & \frac{1}{5^{m}} \sum_{i=0}^{m-1}(-1)^{i}\left(\begin{array}{c}
2 m \\
i
\end{array}\right) \\
& \times\left(\frac{(-1)^{n(i+1)} F_{2(m-i)(n+1)}+(-1)^{(n+1)(i+1)} F_{2(m-i) n}-F_{2(m-i)}}{F_{2(m-i)}}\right)
\end{aligned}
$$




$$
\begin{aligned}
& +\frac{1}{5^{m}}\left(\begin{array}{c}
2 m \\
m
\end{array}\right) \sum_{k=1}^{n}(-1)^{m(k+1)+k} \\
= & \frac{1}{5^{m}} \sum_{i=0}^{m-1}(-1)^{i(n+1)+n}\left(\begin{array}{c}
2 m \\
i
\end{array}\right)\left(\frac{F_{2(m-i)(n+1)}-(-1)^{i} F_{2(m-i) n}}{F_{2(m-i)}}\right) \\
& -\frac{1}{5^{m}} \sum_{i=0}^{m-1}(-1)^{i}\left(\begin{array}{c}
2 m \\
i
\end{array}\right)+\frac{1}{5^{m}}\left(\begin{array}{c}
2 m \\
m
\end{array}\right) \sum_{k=1}^{n}(-1)^{m(k+1)+k} .
\end{aligned}
$$

Using ( $i$ i ) in Lemma 1, we have the claimed result. For even $m$, the desired result is also obtained.

i i) Consider

$$
\begin{aligned}
& \sum_{k=1}^{n}(-1)^{k} F_{k}^{2 m+1} \\
= & \sum_{k=1}^{n}(-1)^{k}\left(\frac{\alpha^{k}-\beta^{k}}{\alpha-\beta}\right)^{2 m+1} \\
= & \sum_{k=1}^{n} \frac{(-1)^{k}}{(\alpha-\beta)^{2 m+1}} \sum_{i=0}^{2 m+1}\left(\begin{array}{c}
2 m+1 \\
i
\end{array}\right)(-1)^{i+1} \alpha^{k i} \beta^{k(2 m+1-i)} \\
= & \sum_{k=1}^{n} \frac{(-1)^{k}}{(\alpha-\beta)^{2 m+1}} \sum_{i=0}^{m}\left(\begin{array}{c}
2 m+1 \\
i
\end{array}\right)(-1)^{i}\left(\alpha^{k(2 m+1-i)} \beta^{k i}-\alpha^{k i} \beta^{k(2 m+1-i)}\right) \\
= & \frac{1}{5^{m}} \sum_{i=0}^{m}(-1)^{i}\left(\begin{array}{c}
2 m+1 \\
i
\end{array}\right) \sum_{k=1}^{n}(-1)^{k(i+1)} F_{k(2 m-2 i+1)} .
\end{aligned}
$$

By taking $r=i+1$ and $t=2(m-i)$ in Corollary 1 , we write

$$
\begin{aligned}
& \sum_{k=1}^{n}(-1)^{k} F_{k}^{2 m+1} \\
= & \frac{1}{5^{m}} \sum_{i=0}^{m}(-1)^{i}\left(\begin{array}{c}
2 m+1 \\
i
\end{array}\right) \times \\
& \frac{(-1)^{(n+1)(i+1)} F_{(2 m-2 i+1) n}+(-1)^{n(i+1)} F_{(2 m-2 i+1)(n+1)}-F_{2 m-2 i+1}}{L_{2 m-2 i+1}} \\
= & \frac{1}{5^{m}} \sum_{i=0}^{m}(-1)^{i+n(i+1)}\left(\begin{array}{c}
2 m+1 \\
i
\end{array}\right) \frac{(-1)^{i+1} F_{(2 m-2 i+1) n}+F_{(2 m-2 i+1)(n+1)}}{L_{2 m-2 i+1}}
\end{aligned}
$$




$$
-\frac{1}{5^{m}} \sum_{i=0}^{m}(-1)^{i}\left(\begin{array}{c}
2 m+1 \\
i
\end{array}\right) \frac{F_{2 m-2 i+1}}{L_{2 m-2 i+1}} .
$$

By Lemma 1, we obtain the claimed result.

For further use, we state a consequence of Lemma 2:

Corollary 2. For even positive integer $t$ and $n>0$,

$$
\sum_{k=1}^{n}(-1)^{k} L_{2 t k}=\frac{(-1)^{n} L_{t(2 n+1)}}{L_{t}}-1 .
$$

Proof. Substituting $a=1, p=2 t$ and $q=0$ in Lemma 2, we get

$$
\begin{aligned}
\sum_{k=1}^{n}(-1)^{k} L_{2 t k} & =\frac{(-1)^{n} L_{2 t(n+1)}+(-1)^{n} L_{2 t n}-L_{2 t}-2}{2+L_{2 t}} \\
& =\frac{(-1)^{n}\left(L_{2 t(n+1)}+L_{2 t n}\right)-\left(L_{2 t}+2\right)}{2+L_{2 t}}
\end{aligned}
$$

For even $t$, from $[1,5]$, we have that

$$
L_{c+t}+L_{c-t}=L_{c} L_{t}
$$

and for any $c$,

$$
L_{2 c}+(-1)^{c} 2=L_{c}^{2}
$$

Thus we obtain

$$
\sum_{k=1}^{n}(-1)^{k} L_{2 t k}=(-1)^{n} \frac{L_{(2 n+1) t}}{L_{t}}-1,
$$

as claimed.

Theorem 2. For $m>0$,

$$
\begin{aligned}
& \text { i) } \quad \sum_{k=1}^{n}(-1)^{k} F_{2 k}^{2 m} \\
& =\left\{\begin{array}{c}
\frac{1}{5^{m}} \sum_{i=0}^{m-1}(-1)^{i}\left(\begin{array}{c}
2 m \\
i
\end{array}\right) \frac{L_{2(m-i)(2 n+1)}}{L_{2(m-i)}}+\frac{(-1)^{m}}{5^{m}}\left(\begin{array}{c}
2 m-1 \\
m-1
\end{array}\right) \\
\frac{1}{5^{m}} \sum_{i=0}^{m-1}(-1)^{i+1}\left(\begin{array}{c}
2 m \\
i
\end{array}\right) \frac{L_{2(m-i)(2 n+1)}}{L_{2(m-i)}}-\frac{(-1)^{m}}{5^{m}}\left(\begin{array}{c}
2 m-1 \\
m-1
\end{array}\right) \quad \text { if } n \text { is oven, },
\end{array}\right.
\end{aligned}
$$

and

$$
\text { ii) } \quad \sum_{k=1}^{n}(-1)^{k} F_{2 k}^{2 m+1}
$$




$$
=\frac{1}{5^{m+1}} \sum_{i=0}^{m}(-1)^{i}\left(\begin{array}{c}
2 m+1 \\
i
\end{array}\right) \frac{(-1)^{n} L_{(2 m-2 i+1)(2 n+1)}-L_{2 m-2 i+1}}{F_{2 m-2 i+1}} .
$$

Proof. i) We write

$$
\begin{aligned}
& \sum_{k=1}^{n}(-1)^{k} F_{2 k}^{2 m}=\sum_{k=1}^{n}(-1)^{k}\left(\frac{\alpha^{2 k}-\beta^{2 k}}{\alpha-\beta}\right)^{2 m} \\
& =\sum_{k=1}^{n}(-1)^{k} \frac{1}{(\alpha-\beta)^{2 m}} \sum_{i=0}^{2 m}(-1)^{i}\left(\begin{array}{c}
2 m \\
i
\end{array}\right) \alpha^{2 k i} \beta^{2 k(2 m-i)} \\
& =\sum_{k=1}^{n} \frac{(-1)^{k}}{5^{m}}\left(\sum_{i=0}^{m-1}(-1)^{i}\left(\begin{array}{c}
2 m \\
i
\end{array}\right)\left(\alpha^{2 k(2 m-i)} \beta^{2 k i}+\alpha^{2 k i} \beta^{2 k(2 m-i)}\right)\right. \\
& \left.+(-1)^{m}\left(\begin{array}{c}
2 m \\
m
\end{array}\right)(\alpha \beta)^{2 k m}\right) \\
& =\sum_{k=1}^{n} \frac{(-1)^{k}}{5^{m}}\left(\sum_{i=0}^{m-1}(-1)^{i}\left(\begin{array}{c}
2 m \\
i
\end{array}\right) L_{4 k(m-i)}+(-1)^{m}\left(\begin{array}{c}
2 m \\
m
\end{array}\right)\right) \\
& =\frac{1}{5^{m}}\left(\sum_{i=0}^{m-1}(-1)^{i}\left(\begin{array}{c}
2 m \\
i
\end{array}\right) \sum_{k=1}^{n}(-1)^{k} L_{4 k(m-i)}+(-1)^{m}\left(\begin{array}{c}
2 m \\
m
\end{array}\right) \sum_{k=1}^{n}(-1)^{k}\right) \text {. }
\end{aligned}
$$

If we take $t=2(m-i)$ in Corollary 2, we write

$$
\begin{aligned}
& \sum_{k=1}^{n}(-1)^{k} F_{2 k}^{2 m} \\
= & \frac{1}{5^{m}} \sum_{i=0}^{m-1}\left(\begin{array}{c}
2 m \\
i
\end{array}\right)(-1)^{i}\left(\frac{(-1)^{n} L_{2(m-i)(2 n+1)}}{L_{2(m-i)}}-1\right) \\
& +\frac{1}{5^{m}}\left(\begin{array}{c}
2 m \\
m
\end{array}\right)(-1)^{m} \sum_{k=1}^{n}(-1)^{k} \\
= & \frac{1}{5^{m}} \sum_{i=0}^{m-1}(-1)^{n+i}\left(\begin{array}{c}
2 m \\
i
\end{array}\right) \frac{L_{2(m-i)(2 n+1)}}{L_{2(m-i)}}-\frac{1}{5^{m}} \sum_{i=0}^{m-1}(-1)^{i}\left(\begin{array}{c}
2 m \\
i
\end{array}\right) \\
& +\frac{1}{5^{m}}\left(\begin{array}{c}
2 m \\
m
\end{array}\right)(-1)^{m} \sum_{k=1}^{n}(-1)^{k} \\
= & \frac{1}{5^{m}} \sum_{i=0}^{m-1}(-1)^{n+i}\left(\begin{array}{c}
2 m \\
i
\end{array}\right) \frac{L_{2(m-i)(2 n+1)}}{L_{2(m-i)}}
\end{aligned}
$$




$$
+\frac{1}{5^{m}}(-1)^{m}\left(\left(\begin{array}{c}
2 m-1 \\
m-1
\end{array}\right)+\left(\begin{array}{c}
2 m \\
m
\end{array}\right)\left(\frac{(-1)^{n}-1}{2}\right)\right) .
$$

According to the choice of $n$ as an odd or as an even number, we have the conclusion.

$i i$ ) The proof is obtained similary to the proof of $(i)$.

For further use, we state the following result:

Corollary 3. For positive even integer $t$ and $n>0$,

$$
\sum_{k=1}^{n}(-1)^{k} L_{(2 k+1) t}=\frac{(-1)^{n} L_{2 t(n+1)}-L_{2 t}}{L_{t}} .
$$

Proof. Substituting $a=1, p=2 t$ and $q=t$ in Lemma 2, we get

$$
\begin{aligned}
\sum_{k=1}^{n}(-1)^{k} L_{(2 k+1) t} & =\frac{(-1)^{n} L_{(2 n+3) t}+(-1)^{n} L_{(2 n+1) t}-L_{3 t}-L_{t}}{2+L_{2 t}} \\
& =\frac{(-1)^{n}\left(L_{(2 n+3) t}+L_{(2 n+1) t}\right)-\left(L_{3 t}+L_{t}\right)}{2+L_{2 t}} .
\end{aligned}
$$

By (2.11) and (2.12), we rewrite the last equation as for even $t$,

$$
\begin{aligned}
\sum_{k=1}^{n}(-1)^{k} L_{(2 k+1) t} & =(-1)^{n} \frac{L_{t} L_{2 t(n+1)}}{L_{t}^{2}}-\frac{L_{3 t}+L_{t}}{L_{t}^{2}} \\
& =(-1)^{n} \frac{L_{2 t(n+1)}}{L_{t}}-\frac{L_{2 t}}{L_{t}} .
\end{aligned}
$$

Thus we have the conclusion.

Theorem 3. For $m>0$,

$$
\begin{aligned}
\text { i) } \sum_{k=1}^{n}(-1)^{k} F_{2 k+1}^{2 m} & \begin{cases}\frac{-1}{5^{m}} \sum_{i=0}^{m-1}\left(\begin{array}{c}
2 m \\
i
\end{array}\right) \frac{L_{4(m-i)(n+1)}+L_{4(m-i)}}{L_{2(m-i)}}-\frac{1}{5^{m}}\left(\begin{array}{c}
2 m \\
m
\end{array}\right) & \text { if } n \text { is odd } \\
\frac{1}{5^{m}} \sum_{i=0}^{m-1}\left(\begin{array}{c}
2 m \\
i
\end{array}\right) \frac{L_{4(m-i)(n+1)}-L_{4(m-i)}}{L_{2(m-i)}} & \text { if } n \text { is even, }\end{cases}
\end{aligned}
$$

and

ii) $\sum_{k=1}^{n}(-1)^{k} F_{2 k+1}^{2 m+1}=\frac{1}{5^{m+1}} \sum_{i=0}^{m}\left(\begin{array}{c}2 m+1 \\ i\end{array}\right) \frac{(-1)^{n} L_{(4 m-4 i+2)(n+1)}-L_{4 m-4 i+2}}{F_{2 m-2 i+1}}$. 
Proof. i) Consider

$$
\begin{aligned}
& \sum_{k=1}^{n}(-1)^{k} F_{2 k+1}^{2 m} \\
& =\sum_{k=1}^{n}(-1)^{k}\left(\frac{\alpha^{2 k+1}-\beta^{2 k+1}}{\alpha-\beta}\right)^{2 m} \\
& =\sum_{k=1}^{n} \frac{(-1)^{k}}{(\alpha-\beta)^{2 m}} \sum_{i=0}^{2 m}(-1)^{i}\left(\begin{array}{c}
2 m \\
i
\end{array}\right) \alpha^{(2 k+1) i} \beta^{(2 k+1)(2 m-i)} \\
& =\sum_{k=1}^{n} \frac{(-1)^{k}}{5^{m}}\left(\sum_{i=0}^{m-1}(-1)^{i}\left(\begin{array}{c}
2 m \\
i
\end{array}\right)\right. \\
& \left(\alpha^{(2 k+1)(2 m-i)} \beta^{(2 k+1) i}+\alpha^{(2 k+1) i} \beta^{(2 k+1)(2 m-i)}\right) \\
& \left.+\left(\begin{array}{c}
2 m \\
m
\end{array}\right)(\alpha \beta)^{2 k m}\right) \\
& =\sum_{k=1}^{n} \frac{(-1)^{k}}{5^{m}}\left(\sum_{i=0}^{m-1}\left(\begin{array}{c}
2 m \\
i
\end{array}\right) L_{2(2 k+1)(m-i)}+\left(\begin{array}{c}
2 m \\
m
\end{array}\right)\right) \\
& =\frac{1}{5^{m}}\left(\sum_{i=0}^{m-1}\left(\begin{array}{c}
2 m \\
i
\end{array}\right) \sum_{k=1}^{n}(-1)^{k} L_{2(2 k+1)(m-i)}+\left(\begin{array}{c}
2 m \\
m
\end{array}\right) \sum_{k=1}^{n}(-1)^{k}\right)
\end{aligned}
$$

If we take $t=2(m-i)$ in Corollary 3 , we write

$$
\begin{aligned}
& \sum_{k=1}^{n}(-1)^{k} F_{2 k+1}^{2 m} \\
= & \frac{1}{5^{m}} \sum_{i=0}^{m-1}\left(\begin{array}{c}
2 m \\
i
\end{array}\right) \frac{(-1)^{n} L_{4(m-i)(n+1)}-L_{4(m-i)}}{L_{2(m-i)}}+\frac{1}{5^{m}}\left(\begin{array}{c}
2 m \\
m
\end{array}\right) \sum_{k=1}^{n}(-1)^{k} \\
= & \frac{(-1)^{n}}{5^{m}} \sum_{i=0}^{m-1}\left(\begin{array}{c}
2 m \\
i
\end{array}\right) \frac{L_{4(m-i)(n+1)}}{L_{2(m-i)}} \\
& -\frac{1}{5^{m}} \sum_{i=0}^{m-1}\left(\begin{array}{c}
2 m \\
i
\end{array}\right) \frac{L_{4(m-i)}}{L_{2(m-i)}}+\frac{1}{5^{m}}\left(\begin{array}{c}
2 m \\
m
\end{array}\right)\left(\frac{(-1)^{n}-1}{2}\right) .
\end{aligned}
$$

According to the chice of $n$ as an odd or as an even number, we have the conclusion. $i i$ ) The proof of $(i i)$ is obtained similary to the proof of $(i)$. 


\section{Alternating Melham's SUm fOR LuCAS NUmbers}

Theorem 4. i) For positive odd $m$,

$$
\begin{array}{r}
\sum_{k=1}^{n}(-1)^{k} L_{k}^{2 m}=\sum_{i=0}^{m-1}(-1)^{(i+1) n}\left(\begin{array}{c}
2 m \\
i
\end{array}\right) \\
+\left(\begin{array}{c}
2 m-1 \\
m
\end{array}\right)+\left(\begin{array}{c}
2 m \\
m
\end{array}\right) n,
\end{array}
$$

and, for even $m$,

$$
\begin{aligned}
& \sum_{k=1}^{n}(-1)^{k} L_{k}^{2 m} \\
= & \left\{\begin{array}{c}
\sum_{i=0}^{m-1}\left(\begin{array}{c}
2 m \\
i
\end{array}\right) \frac{L_{(m-i)(2 n+1)}}{L_{m-i}}-2^{2 m-1}+\left(\begin{array}{c}
2 m-1 \\
m
\end{array}\right) \quad \text { if } n \text { is even, } \\
\sum_{i=0}^{m-1}(-1)^{i+1}\left(\begin{array}{c}
2 m \\
i
\end{array}\right) \frac{L_{(m-i)(2 n+1)}}{L_{m-i}}-2^{2 m-1} \\
+\left(\begin{array}{c}
2 m-1 \\
m
\end{array}\right)-\left(\begin{array}{c}
2 m \\
m
\end{array}\right)
\end{array} \quad \text { if } n\right. \text { is odd, }
\end{aligned}
$$

and

ii) $\sum_{k=1}^{n}(-1)^{k} L_{k}^{2 m+1}=\sum_{i=0}^{m}\left(\begin{array}{c}2 m+1 \\ i\end{array}\right) \frac{(-1)^{(i+1) n+1}}{L_{2 m-2 i+1}}$

$$
\begin{aligned}
\times \sum_{j=0}^{2(m-i)}(-1)^{j i+1} & L_{n(2 m-2 i+1)+j+\frac{1-3(-1)^{i}}{2}} \\
+ & 2 \sum_{i=0}^{m}(-1)^{i}\left(\begin{array}{c}
2 m+1 \\
i
\end{array}\right) \frac{1}{L_{2 m-2 i+1}}-2^{2 m} .
\end{aligned}
$$

Proof. $i$ ) By the Binet formula of $\left\{L_{n}\right\}$, we have

$$
\begin{aligned}
& \sum_{k=1}^{n}(-1)^{k} L_{k}^{2 m}=\sum_{k=1}^{n}(-1)^{k}\left(\alpha^{k}+\beta^{k}\right)^{2 m} \\
= & \sum_{k=1}^{n}(-1)^{k}\left(\sum_{i=0}^{m-1}\left(\begin{array}{c}
2 m \\
i
\end{array}\right)\left(\alpha^{k(2 m-i)} \beta^{k i}+\alpha^{k i} \beta^{k(2 m-i)}\right)+\left(\begin{array}{c}
2 m \\
m
\end{array}\right)(\alpha \beta)^{k m}\right) .
\end{aligned}
$$


Since $\alpha \beta=-1$, we get

$$
\sum_{k=1}^{n}(-1)^{k} L_{k}^{2 m}=\sum_{i=0}^{m-1}\left(\begin{array}{c}
2 m \\
i
\end{array}\right) \sum_{k=1}^{n}(-1)^{k(i+1)} L_{2 k(m-i)}+\left(\begin{array}{c}
2 m \\
m
\end{array}\right) \sum_{k=1}^{n}(-1)^{k(m+1)} .
$$

From Corollary 1, we write

$$
\begin{aligned}
& \sum_{k=1}^{n}(-1)^{k} L_{k}^{2 m} \\
& =\sum_{i=0}^{m-1}\left(\begin{array}{c}
2 m \\
i
\end{array}\right) \\
& \left(\frac{(-1)^{(i+1) n} F_{2(m-i)(n+1)}+(-1)^{(n+1)(i+1)} F_{2(m-i) n}-F_{2(m-i)}}{F_{2(m-i)}}\right) \\
& +\left(\begin{array}{c}
2 m \\
m
\end{array}\right) \sum_{k=1}^{n}(-1)^{k(m+1)} \\
& =\sum_{i=0}^{m-1}(-1)^{(i+1) n}\left(\begin{array}{c}
2 m \\
i
\end{array}\right) \frac{F_{2(m-i)(n+1)}+(-1)^{i+1} F_{2(m-i) n}}{F_{2(m-i)}} \\
& -\sum_{i=0}^{m-1}\left(\begin{array}{c}
2 m \\
i
\end{array}\right)+\left(\begin{array}{c}
2 m \\
m
\end{array}\right) \sum_{k=1}^{n}(-1)^{k(m+1)} \\
& \int \sum_{i=0}^{m-1}(-1)^{(i+1) n}\left(\begin{array}{c}
2 m \\
i
\end{array}\right) \frac{F_{(m-i)(2 n+1)}}{F_{m-i}}-2^{2 m-1} \\
& +\left(\begin{array}{c}
2 m-1 \\
m
\end{array}\right)+\left(\begin{array}{c}
2 m \\
m
\end{array}\right) n \\
& =\left\{\begin{array}{l}
\sum_{i=0}^{m-1}(-1)^{(i+1) n}\left(\begin{array}{c}
2 m \\
i
\end{array}\right) \frac{L_{(m-i)(2 n+1)}}{L_{m-i}}-2^{2 m-1} \\
\text { (2m }
\end{array}\right. \\
& +\left(\begin{array}{c}
2 m-1 \\
m
\end{array}\right)+\left(\begin{array}{c}
2 m \\
m
\end{array}\right)\left(\frac{(-1)^{n}-1}{2}\right)
\end{aligned}
$$

According to the choice of $n$, the claim is obtained.

$i i$ ) The proof is similar to the proof of $i$ ).

Theorem 5. i) For $m>0$ and even $n>0$,

$$
\sum_{k=1}^{n}(-1)^{k} L_{2 k}^{2 m}=\sum_{i=0}^{m-1}\left(\begin{array}{c}
2 m \\
i
\end{array}\right) \frac{L_{2(m-i)(2 n+1)}}{L_{2(m-i)}}-2^{2 m-1}+\left(\begin{array}{c}
2 m-1 \\
m
\end{array}\right)
$$


and, for odd $n>0$,

$$
\sum_{k=1}^{n}(-1)^{k} L_{2 k}^{2 m}=-\sum_{i=0}^{m-1}\left(\begin{array}{c}
2 m \\
i
\end{array}\right) \frac{L_{2(m-i)(2 n+1)}}{L_{2(m-i)}}-2^{2 m-1}-\left(\begin{array}{c}
2 m-1 \\
m
\end{array}\right) .
$$

ii) For positive integers $m$ and $n$,

$$
\sum_{k=1}^{n}(-1)^{k} L_{2 k}^{2 m+1}=(-1)^{n} \sum_{i=0}^{m}\left(\begin{array}{c}
2 m+1 \\
i
\end{array}\right) \frac{F_{(2 m-2 i+1)(2 n+1)}}{F_{2 m-2 i+1}}-2^{2 m} .
$$

Proof. i) We write

$$
\begin{aligned}
& \sum_{k=1}^{n}(-1)^{k} L_{2 k}^{2 m} \\
= & \sum_{k=1}^{n}(-1)^{k}\left(\alpha^{2 k}+\beta^{2 k}\right)^{2 m} \\
= & \sum_{k=1}^{n}(-1)^{k} \\
& \left(\sum_{i=0}^{m-1}\left(\begin{array}{c}
2 m \\
i
\end{array}\right)\left(\alpha^{2 k(2 m-i)} \beta^{2 k i}+\alpha^{2 k i} \beta^{2 k(2 m-i)}\right)+\left(\begin{array}{c}
2 m \\
m
\end{array}\right)(\alpha \beta)^{2 k m}\right) \\
= & \sum_{i=0}^{m-1}\left(\begin{array}{c}
2 m \\
i
\end{array}\right) \sum_{k=1}^{n}(-1)^{k} L_{4 k(m-i)}+\left(\begin{array}{c}
2 m \\
m
\end{array}\right) \sum_{k=1}^{n}(-1)^{k} .
\end{aligned}
$$

If we take $t=2(m-i)$ in Corollary 2, we can write

$$
\begin{aligned}
& \sum_{k=1}^{n}(-1)^{k} L_{2 k}^{2 m} \\
= & \sum_{i=0}^{m-1}\left(\begin{array}{c}
2 m \\
i
\end{array}\right)\left(\frac{(-1)^{n} L_{2(m-i)(2 n+1)}}{L_{2(m-i)}}-1\right)+\left(\begin{array}{c}
2 m \\
m
\end{array}\right) \sum_{k=1}^{n}(-1)^{k} \\
= & (-1)^{n} \sum_{i=0}^{m-1}\left(\begin{array}{c}
2 m \\
i
\end{array}\right) \frac{L_{2(m-i)(2 n+1)}}{L_{2(m-i)}}-\sum_{i=0}^{m-1}\left(\begin{array}{c}
2 m \\
i
\end{array}\right)+\left(\begin{array}{c}
2 m \\
m
\end{array}\right) \sum_{k=1}^{n}(-1)^{k} \\
= & (-1)^{n} \sum_{i=0}^{m-1}\left(\begin{array}{c}
2 m \\
i
\end{array}\right) \frac{L_{2(m-i)(2 n+1)}}{L_{2(m-i)}}-2^{2 m-1}+\left(\begin{array}{c}
2 m-1 \\
m-1
\end{array}\right)+\left(\begin{array}{c}
2 m \\
m
\end{array}\right)\left(\frac{(-1)^{n}-1}{2}\right) .
\end{aligned}
$$


According to the choice of $n$, the proof is complete by the fact that

$$
\left(\begin{array}{l}
n \\
k
\end{array}\right)=\left(\begin{array}{l}
n-1 \\
k-1
\end{array}\right)+\left(\begin{array}{c}
n-1 \\
k
\end{array}\right) .
$$

ii) The proof of $(i i)$ is similar to the proof of $(i)$.

Theorem 6. For $m>0$,

$$
\begin{aligned}
& \text { i) } \sum_{k=1}^{n}(-1)^{k} L_{2 k+1}^{2 m} \\
& = \begin{cases}\sum_{i=0}^{m-1}(-1)^{i}\left(\begin{array}{c}
2 m \\
i
\end{array}\right) \frac{L_{4(m-i)(n+1)}-L_{4(m-i)}}{L_{2(m-i)}} & \text { if } n \text { is even, } \\
(-1)^{m+1}\left(\begin{array}{c}
2 m \\
m
\end{array}\right)-\sum_{i=0}^{m-1}(-1)^{i}\left(\begin{array}{c}
2 m \\
i
\end{array}\right) \frac{L_{4(m-i)(n+1)}+L_{4(m-i)}}{L_{2(m-i)}} & \text { if } n \text { is odd, }\end{cases}
\end{aligned}
$$

and

$$
\begin{gathered}
\text { ii) } \sum_{k=1}^{n}(-1)^{k} L_{2 k+1}^{2 m+1}= \\
\sum_{i=0}^{m}(-1)^{i}\left(\begin{array}{c}
2 m+1 \\
i
\end{array}\right) \frac{(-1)^{n} F_{2(2 m-2 i+1)(n+1)}-F_{2(2 m-2 i+1)}}{F_{2 m-2 i+1}} .
\end{gathered}
$$

Proof. $i$ ) Using the Binet formula of $\left\{L_{n}\right\}$, we have

$$
\begin{aligned}
& \sum_{k=1}^{n}(-1)^{k} L_{2 k+1}^{2 m} \\
= & \sum_{k=1}^{n}(-1)^{k}\left(\alpha^{2 k+1}+\beta^{2 k+1}\right)^{2 m} \\
= & \sum_{k=1}^{n}(-1)^{k}\left(\sum_{i=0}^{m-1}\left(\begin{array}{c}
2 m \\
i
\end{array}\right)\left(\alpha^{(2 k+1)(2 m-i)} \beta^{(2 k+1) i}+\alpha^{(2 k+1) i} \beta^{(2 k+1)(2 m-i)}\right)\right. \\
& \left.+\left(\begin{array}{c}
2 m \\
m
\end{array}\right)(\alpha \beta)^{(2 k+1) m}\right) \\
= & \sum_{k=1}^{n}(-1)^{k}\left(\sum_{i=0}^{m-1}(-1)^{i}\left(\begin{array}{c}
2 m \\
i
\end{array}\right)\left(\alpha^{(2 k+1)(2 m-2 i)}+\beta^{(2 k+1)(2 m-2 i)}\right)\right.
\end{aligned}
$$




$$
\begin{aligned}
& \left.+\left(\begin{array}{c}
2 m \\
m
\end{array}\right)(-1)^{m}\right) \\
= & \sum_{i=0}^{m-1}(-1)^{i}\left(\begin{array}{c}
2 m \\
i
\end{array}\right) \sum_{k=1}^{n}(-1)^{k} L_{2(2 k+1)(m-i)}+\left(\begin{array}{c}
2 m \\
m
\end{array}\right)(-1)^{m} \sum_{k=1}^{n}(-1)^{k} .
\end{aligned}
$$

If we take $t=2(m-i)$ in Corollary 3 , we get

$$
\begin{aligned}
& \sum_{k=1}^{n}(-1)^{k} L_{2 k+1}^{2 m} \\
= & \sum_{i=0}^{m-1}(-1)^{i}\left(\begin{array}{c}
2 m \\
i
\end{array}\right)\left(\frac{(-1)^{n} L_{4(m-i)(n+1)}-L_{4(m-i)}}{L_{2(m-i)}}\right) \\
& +\left(\begin{array}{c}
2 m \\
m
\end{array}\right)(-1)^{m} \sum_{k=1}^{n}(-1)^{k} \\
= & \sum_{i=0}^{m-1}(-1)^{i}\left(\begin{array}{c}
2 m \\
i
\end{array}\right) \frac{(-1)^{n} L_{4(m-i)(n+1)}}{L_{2(m-i)}}-\sum_{i=0}^{m-1}(-1)^{i}\left(\begin{array}{c}
2 m \\
i
\end{array}\right) \frac{L_{4(m-i)}}{L_{2(m-i)}} \\
& +\left(\begin{array}{c}
2 m \\
m
\end{array}\right)(-1)^{m} \sum_{k=1}^{n}(-1)^{k} \\
= & (-1)^{n} \sum_{i=0}^{m-1}(-1)^{i}\left(\begin{array}{c}
2 m \\
i
\end{array}\right) \frac{L_{4(m-i)(n+1)}}{L_{2(m-i)}}-\sum_{i=0}^{m-1}(-1)^{i}\left(\begin{array}{c}
2 m \\
i
\end{array}\right) \frac{L_{4(m-i)}}{L_{2(m-i)}} \\
& +\left(\begin{array}{c}
2 m \\
m
\end{array}\right)(-1)^{m}\left(\frac{(-1)^{n}-1}{2}\right) .
\end{aligned}
$$

According to the choice of $n$, the claimed result is clear.

$i i$ ) The proof is similar to the proof of $i$ ).

\section{REFERENCES}

[1] T. Koshy, Fibonacci and Lucas numbers with applications, ser. Pure and Applied Mathematics (New York). New York: Wiley-Interscience, 2001.

[2] K. Ozeki, “On Melham's sum,” Fibonacci Quart., vol. 46/47, no. 2, pp. 107-110, 2008/09.

[3] H. Prodinger, "On a sum of Melham and its variants," Fibonacci Quart., vol. 46/47, no. 3, pp. 207-215, 2008/09.

[4] J. Seibert and P. Trojovský, "On sums of certain products of Lucas numbers," Fibonacci Quart., vol. 44, no. 2, pp. 172-180, 2006.

[5] S. Vajda, "Fibonacci \& Lucas numbers, and the golden section," in Theory and applications, ser. Ellis Horwood Series: Mathematics and its Applications. New York: John Wiley \& Sons, Inc., 1989, p. 190. 
[6] M. Wiemann and C. Cooper, "Divisibility of an $F-L$ type convolution," in Applications of Fibonacci numbers, ser. Proceedings of the 10th International Research Conference on Fibonacci Numbers and their Applications held at Northern Arizona University, vol. 9. Dordrecht: Kluwer Acad. Publ., 2004, pp. 267-287.

Authors' addresses

\section{Emrah Kılıç}

TOBB Economics and Technology University Mathematics Department 06560 Ankara Turkey

E-mail address: ekilicdetu.edu.tr

\section{Neşe Ömür}

Kocaeli University Mathematics Department 41380 Izmit Turkey

E-mail address: neseomur@kocaeli.edu.tr

Yücel Türker Ulutaş

Kocaeli University Mathematics Department 41380 Izmit Turkey

E-mail address: turkery@kocaeli.edu.tr 